\title{
Compact North Finding System
}

\section{Citation}

Collin, J., Takala, J., Bojja, J., Kirkko-Jaakkola, M., Payne, M., \& Griffiths, R. (2016). Compact North Finding System. IEEE Sensors Journal, 16(8), 2554-2563. DOI: 10.1109/JSEN.2016.2518860

Year

2016

\section{Version}

Peer reviewed version (post-print)

\section{Link to publication}

TUTCRIS Portal (http://www.tut.fi/tutcris)

\author{
Published in \\ IEEE Sensors Journal
}

\section{DOI}

10.1109/JSEN.2016.2518860

\section{Copyright}

(C) 2016 IEEE. Personal use of this material is permitted. Permission from IEEE must be obtained for all other uses, in any current or future media, including reprinting/republishing this material for advertising or promotional purposes, creating new collective works, for resale or redistribution to servers or lists, or reuse of any copyrighted component of this work in other works.

\section{Take down policy}

If you believe that this document breaches copyright, please contact tutcris@tut.fi, and we will remove access to the work immediately and investigate your claim. 


\title{
Compact North Finding System
}

\author{
Jayaprasad Bojja, Jussi Collin, Martti Kirkko-Jaakkola*, Martin Payne ${ }^{\dagger}$, Ryan Griffiths ${ }^{\dagger}$ and Jarmo Takala \\ Dept. Pervasive Computing, Tampere University of Technology, Finland. \\ *Finnish Geospatial Research Institute, Kirkkonummi, Finland. \\ $\dagger$ Geovista Ltd, Glan Conwy, UK.
}

\begin{abstract}
The knowledge of orientation of an object with respect to earth-fixed reference coordinate system is crucial in many applications. For instance, in oil mining it is very crucial to accurately know the orientation of the drilling equipment under the earth surface to drill through desired path. In this context we propose a compact inertial sensor system that estimates the instantaneous orientation of the system using accelerometer and gyroscope-derived tilt and azimuth angles. To keep the system size small, we use two-axis accelerometer and one-axis gyroscope. In addition, to avoid high sensor cost, the sensor biases are removed using indexing method. The proposed system estimates the orientation of the compact system in almost all of the orientations and additionally it also provides the measurement accuracy and integrity values that help in ascertaining the validity of the orientation estimate.
\end{abstract}

Index Terms-MEMS Accelerometer, Optical fiber gyroscope sensor, Azimuth, Least square approximations, Sonde, North Seeking.

\section{INTRODUCTION}

$\mathbf{S}$ ENSOR systems combining accelerometers-based tilt and magnetometer-based azimuth are typically used in instantaneous orientation estimation when the size and cost of the system is limited. Such systems are inherently capable of estimating orientation w.r.t magnetic north and will require corrections to align with true north, and presence of ferromagnetic materials in the vicinity of such systems can corrupt the solution. To overcome problems related to magnetic field sensing, other types of sensors, such as Sun sensors [1], and gyroscopes sensors [2], [3] have been proposed to be used in the system. These systems are built to be self-contained systems, i.e, they do not require initial attitude information as input. Instead these systems estimates the attitude information discontinuously, on demand basis and under stationary conditions, unlike [4], [5] where in, knowledge of the initial attitude is essential in order to track the orientation continuously w.r.t initial attitude and are non-stationary in nature. In these north finding systems, preferably, gyroscopes capable of sensing the Earth's rotation rate are often used, as they provide more self-contained measurements. The Earth's rotation rate is only about $15^{\circ} / \mathrm{hr}$ and measuring of this rate accurately and directly would require large and costly sensor assembly, such as navigation grade inertial navigation system [6]. To

Authors Jayaprasad Bojja, Jussi Collin and Jarmo Takala are with the Department of Pervasive Computing, Tampere University of Technology, Finland. Author Martti Kirkko-Jaakkola is with Finnish Geospatial Research Institute, Masala, Finland. Authors Martin Payne and Ryan Griffiths are with Geovista Ltd, Glan Convy, UK. E-mail: @tut.fi, @nls.fi, @ geovista.co.uk. reduce the requirements for such gyro accuracy, sensor rotation techniques have been proposed through the decades [2], [7], [8]. Recent studies show that indeed the small sized fiber optic gyros [3] or MEMS gyros [9] can be used for this purpose, but with apparently limited tilt angles. This is due to the fact that only uni-axial rotation is applied. A motoring system that could rotate the sensors around many axes would be complex and bulky for many purposes. In [10] North seeking system with uni-axial fiber optic gyro (FOG) and a uni-axial accelerometer is presented. This method estimates the tilt angles by modeling mathematical equations in which the components of residual errors are eliminated. The azimuth accuracy shown in their experiments was at sub-degree level, but with reported tilt angles below 5 degrees.

The limitations of most of the above proposed methods are that, for a roll or pitch angles higher than about 5 degrees, the accuracy of azimuth estimate deteriorates proportionally to the roll and pitch angles. This is because those algorithms are designed to be used only when the rate gyro sensor is strapped down and sensing axis is perpendicular to the horizontal plane rather than in an arbitrary angle. In this context, we propose a compact system capable of estimating orientation for almost all the tilt angles. The system is compact, based on the single 2-axis MEMS accelerometer and a single 1axis FOG rate sensor. In this publication temperature and vibration effects to the sensors is considered to be minimal as the measurement and estimation time in the proposed method is under 2 minutes and under stationary conditions. In real system implementations, such externally influencing factors would be handled appropriately by the manufacturers and additionally during the field applications. In our method, indexing is performed with uni-axial motor system using sequence of 90 degree turns to eliminate sensor biases and errors. The paper is organized as follows. In section II, we elaborate the basics and the implementation of the whole system, and in section III we discuss about the experimental setup, results and discuss briefly about the results. Section IV concludes the paper with the contributions and results of our research work.

\section{ORIENTATION ESTIMATION}

The proposed method relies on the output measurements of both gyroscope and accelerometer. Measurements of gyroscope and accelerometer can be be modeled as

$$
\begin{gathered}
\hat{\omega}=M_{\omega} \omega_{I B}^{B}+\epsilon_{\omega} \\
\hat{a}=M_{a} a_{s f}^{B}+\epsilon_{a}
\end{gathered}
$$


where $M_{\omega}$ and $M_{a}$ are the cross-coupling and scale factor error matrix of gyroscope and accelerometer respectively, $\omega_{I B}^{B}$ is the measured angular rate vector $\left[m_{w x}, m_{w y}, m_{w z}\right]^{T}$, $a_{s f}^{B}$ is the measured specific force vector $\left[m_{a x}, m_{a y}, m_{a z}\right]^{T}$. ()$^{B}$ indicates that the measurements are measured with respect to sensor body frame and ()$_{I B}$ indicates that the measurements are in sensor body coordinate frame relative to inertial frame. Additive measurement error $\epsilon$ typically includes slowly varying bias and uncorrelated (white) noise. Assuming ideal sensors the measurements from stationary (Earth-fixed) unit would be

$$
\begin{aligned}
& \omega_{I B}^{B}=C_{L}^{B} \omega_{I E}^{L} \\
& a_{s f}^{B}=-C_{L}^{B} g_{p}^{L}
\end{aligned}
$$

where $\omega_{I E}^{L}$ is the vector of local rotation rate of Earth, given as

$$
\omega_{I E}^{L}=\left(\begin{array}{c}
\Omega \cos (\lambda) \\
0 \\
\Omega \sin (\lambda)
\end{array}\right)
$$

and $g_{p}^{L}$ is the local plumb-bob gravity [6] vector, given as

$$
g_{p}^{L}=\left(\begin{array}{c}
0 \\
0 \\
-g
\end{array}\right)
$$

where $g$ is the gravity, $\Omega$ is the magnitude of Earth rotation rate, and $\lambda$ is the latitude. The problem in orientation estimation is to find the direction cosine matrix (DCM) $C_{L}^{B}$, that transforms vectors from local level frame to body frame. In this form the problem to be solved would be traditional Wahba's problem [11], which can be easily solved using singular value decomposition, for example [12]. Under stationary and ideal conditions, with 3D accelerometer, and 3D gyroscope measurements in the absence of any kind of noise, using normalized (3), (4), (5), and (6) we can deduce as in [13] or [14], the required DCM as

$$
C_{L}^{B}=\left(\begin{array}{ccc}
\frac{\bar{m}_{w x}-\left(\bar{m}_{a x} \sin (\lambda)\right)}{\cos (\lambda)} & \frac{\bar{m}_{w y}-\left(\bar{m}_{a y} \sin (\lambda)\right)}{\cos (\lambda)} & \frac{\bar{m}_{w z}-\left(\bar{m}_{a z} \sin (\lambda)\right)}{\cos (\lambda)} \\
\frac{\left(\bar{m}_{a y} \bar{m}_{w z}-\bar{m}_{a z} \bar{m}_{w y}\right)}{\cos (\lambda)} & \frac{-\left(\bar{m}_{a x} \bar{m}_{w z}-\bar{m}_{a z} \bar{m}_{w x}\right)}{\cos (\lambda)} & \frac{\left(\bar{m}_{a x}\right.}{\left.\bar{m}_{a x} \bar{m}_{w y}-\bar{m}_{a y} \bar{m}_{w x}\right)} \\
\bar{m}_{a y} & \bar{m}_{a z}
\end{array}\right),
$$

where $\left[\bar{m}_{a x}, \bar{m}_{a y}, \bar{m}_{a z}\right]^{T}$ is the normalized vector of $a_{s f}^{B}$, and $\left[\bar{m}_{w x}, \bar{m}_{w y}, \bar{m}_{w z}\right]^{T}$ is the normalized vector of $\omega_{I B}^{B}$.

However DCM in (7) does not not produce valid DCM with real noisy sensor measurements. But we will later in section II-B, enlighten the usefulness of this DCM in constructing appropriate DCM with the Euler angles estimated by the proposed algorithm. More over, to limit the cost and size of the unit we use a 2D MEMS accelerometer and a 1D fiber optic gyroscope, both having significant bias errors in the measurements. To mitigate the effect of biases as explained in sub section $\mathrm{D}$, we add indexing motor that can rotate the sensor axes along fixed body-frame axis. Thus, the $C_{L}^{B}$ has to be solved for the following equations of our proposed method.

$$
\begin{gathered}
\hat{\omega}_{y}=\left[\begin{array}{lll}
0 & 1 & 0
\end{array}\right] C_{B}^{B n} C_{L}^{B} \omega_{I E}^{L}+\epsilon_{\omega y} \\
{\left[\begin{array}{ll}
\hat{a}_{x} & \hat{a}_{y}
\end{array}\right]^{T}=\left[\begin{array}{lll}
1 & 1 & 0
\end{array}\right] C_{B}^{B n} C_{L}^{B}\left(-g_{p}^{L}\right)+\left[\begin{array}{ll}
\epsilon_{a_{x}} & \epsilon_{a_{y}}
\end{array}\right]^{T}}
\end{gathered}
$$

where $C_{B}^{B n}$ is the coordinate transformation resulting from indexing motor, for $n=0,1,2,3$ positions. Sensor noise terms $\epsilon_{\omega y}, \epsilon_{a_{x}}, \epsilon_{a_{y}}$ contain constant bias part that can be many orders of magnitude larger than required orientation accuracy would permit. It is well known that at least two linearly independent reference vectors are required to determine attitude uniquely [12]. Thus, the role of indexing motor that generates $C_{B}^{B n}$ is twofold; the constant biases must be mitigated (canceled) and to generate sufficient number of observations.

\section{A. Euler Angle Observations}

Our approach to solve $C_{L}^{B}$ in ( 8) and (9), is using $C_{B}^{B n}$, and based on estimation of Euler angles. We define local frame axes as $x \rightarrow$ North, $y \rightarrow$ West, $z \rightarrow U p$ and Local to Body frame DCM for known Euler angles $\phi, \theta, \psi$ is defined as [15]:

$$
C_{L}^{B}=\left(\begin{array}{ccc}
\cos (\psi) \cos (\theta) & \cos (\theta) \sin (\psi) & -\sin (\theta) \\
\cos (\psi) \sin (\phi) \sin (\theta)-\cos (\phi) \sin (\psi) & \cos (\phi) \cos (\psi)+\sin (\phi) \sin (\psi) \sin (\theta) & \cos (\theta) \sin (\phi) \\
\sin (\phi) \sin (\psi)+\cos (\phi) \cos (\psi) \sin (\theta) & \cos (\phi) \sin (\psi) \sin (\theta)-\cos (\psi) \sin (\phi) & \cos (\phi) \cos (\theta)
\end{array}\right)
$$

Using ( 10) accelerometer observations are then

$$
a^{B}=\left(\begin{array}{c}
-g \sin (\theta) \\
g \cos (\theta) \sin (\phi) \\
g \cos (\phi) \cos (\theta)
\end{array}\right)
$$

and gyro observations

$$
\omega^{B}=\left(\begin{array}{c}
\Omega \cos (\lambda) \cos (\psi) \cos (\theta)-\Omega \sin (\lambda) \sin (\theta) \\
\Omega \cos (\theta) \sin (\lambda) \sin (\phi)-\Omega \cos (\lambda)(\cos (\phi) \sin (\psi)-\cos (\psi) \sin (\phi) \sin (\theta)) \\
\Omega \cos (\lambda)(\sin (\phi) \sin (\psi)+\cos (\phi) \cos (\psi) \sin (\theta))+\Omega \cos (\phi) \cos (\theta) \sin (\lambda)
\end{array}\right)
$$

In addition, indexing is performed by rotating positive 90 degree rotations along the body z-axis,

$$
\begin{aligned}
C_{B}^{B 1} & =\left(\begin{array}{ccc}
0 & -1 & 0 \\
1 & 0 & 0 \\
0 & 0 & 1
\end{array}\right) \\
C_{B}^{B 2} & =\left(\begin{array}{ccc}
-1 & 0 & 0 \\
0 & -1 & 0 \\
0 & 0 & 1
\end{array}\right) \\
C_{B}^{B 3} & =\left(\begin{array}{ccc}
0 & 1 & 0 \\
-1 & 0 & 0 \\
0 & 0 & 1
\end{array}\right)
\end{aligned}
$$

yielding indexed accelerometer measurements:

$$
\begin{aligned}
a^{B 1} & =\left(\begin{array}{c}
-g \cos (\theta) \sin (\phi) \\
-g \sin (\theta) \\
g \cos (\phi) \cos (\theta)
\end{array}\right) \\
a^{B 2} & =\left(\begin{array}{c}
g \sin (\theta) \\
-g \cos (\theta) \sin (\phi) \\
g \cos (\phi) \cos (\theta)
\end{array}\right) \\
a^{B 3} & =\left(\begin{array}{c}
g \cos (\theta) \sin (\phi) \\
g \sin (\theta) \\
g \cos (\phi) \cos (\theta)
\end{array}\right)
\end{aligned}
$$

and indexed gyro measurements:

$$
\begin{aligned}
& \omega^{B 1}=\left(\begin{array}{c}
\Omega \cos (\lambda)(\cos (\phi) \sin (\psi)-\sin (\phi) \cos (\psi) \sin (\theta))-\Omega \cos (\theta) \sin (\lambda) \sin (\phi) \\
\Omega \cos (\lambda) \cos (\psi) \cos (\theta)-\Omega \sin (\lambda) \sin (\theta) \\
\Omega \cos (\lambda)(\sin (\phi) \sin (\psi)+\cos (\phi) \cos (\psi) \sin (\theta))+\Omega \cos (\phi) \cos (\theta) \sin (\lambda)
\end{array}\right) \\
& \omega^{B 2}=\left(\begin{array}{c}
\Omega \sin (\lambda) \sin (\theta)-\Omega \cos (\lambda) \cos (\theta) \cos (\psi) \\
\Omega \cos (\lambda)(\cos (\phi) \sin (\psi)-\sin (\phi) \cos (\psi) \sin (\theta))-\Omega \cos (\theta) \sin (\lambda) \sin (\phi) \\
\Omega \cos (\lambda)(\sin (\phi) \sin (\psi)+\cos (\phi) \cos (\psi) \sin (\theta))+\Omega \cos (\phi) \cos (\theta) \sin (\lambda)
\end{array}\right)
\end{aligned}
$$




$$
\omega^{B 3}=\left(\begin{array}{c}
\Omega \cos (\theta) \sin (\lambda) \sin (\phi)-\Omega \cos (\lambda)(\cos (\phi) \sin (\psi)-\cos (\psi) \sin (\phi) \sin (\theta)) \\
\Omega \sin (\lambda) \sin (\theta)-\Omega \cos (\lambda) \cos (\theta) \cos (\psi) \\
\Omega \cos (\lambda)(\sin (\phi) \sin (\psi)+\cos (\phi) \cos (\psi) \sin (\theta))+\Omega \cos (\phi) \cos (\theta) \sin (\lambda)
\end{array}\right)
$$

We later in section II-D, enlighten the role of $C_{B}^{B n}$ in removal of the constant bias effects. The other task of indexing for obtaining sufficient number of independent vectors is not directly fulfilled as longitudinal (body z-axis) measurements are not available. Due to this, solution to some orientations needs to be addressed by additional logical conditioning, as detailed in following section.

\section{B. Algorithm}

We use Gauss-Newton method in our algorithm, which aims to solve and estimate unknowns $x=\theta, \phi, \psi$ the Euler angles iteratively. The method uses current estimate $\hat{x}=\hat{\theta}, \hat{\phi}, \hat{\psi}$ to compute $h(\hat{x})$ : the predicted measurements. Here $h(x)$ is defined as $h(x)=\left[h_{1} h_{2} h_{3} h_{4}\right]^{T}$ where

$$
\begin{gathered}
h_{1}=(-g \sin (\theta)), \\
h_{2}=(g \cos (\theta) \sin (\phi)), \\
h_{3}=(\Omega \cos (\lambda) \cos (\psi) \cos (\theta)-\Omega \sin (\lambda) \sin (\theta)), \\
h_{4}=(\Omega \cos (\theta) \sin (\lambda) \sin (\phi)-\Omega \cos (\lambda)(\cos (\phi) \sin (\psi)-\cos (\psi) \sin (\phi) \sin (\theta))) .
\end{gathered}
$$

Each iteration of the algorithm is performed after the data from all index positions $(0,1,2,3)$ is available.For each iteration, assuming that the current estimate $\hat{x}$ is correct, the method tries to fit this predicted measurements to the actual measurements.

Jacobian matrix $J_{h}$ of $h(x)$ with respect to each unknowns $\phi, \theta, \psi$ is then $J_{h}=\left[\begin{array}{llll}J_{h 1} & J_{h 2} & J_{h 3} & J_{h 4}\end{array}\right]^{T}$, where

$$
\begin{aligned}
& J_{h 1}=\left(\begin{array}{lll}
-g \cos (\theta) & 0 & 0
\end{array}\right), \\
& J_{h 2}=\left(\begin{array}{lll}
-g \sin (\phi) \sin (\theta) & g \cos (\phi) \cos (\theta) & 0
\end{array}\right) \text {, } \\
& J_{h 3}=\left(\begin{array}{c}
-\Omega \cos (\theta) \sin (\lambda)-\Omega \cos (\lambda) \cos (\psi) \sin (\theta) \\
0 \\
-\Omega \cos (\lambda) \cos (\theta) \sin (\psi)
\end{array}\right)^{T} \\
& J_{h 4}=\left(\begin{array}{c}
\Omega \cos (\lambda) \cos (\psi) \cos (\theta) \sin (\phi)-\Omega \sin (\lambda) \sin (\phi) \sin (\theta) \\
\Omega \cos (\lambda)(\sin (\phi) \sin (\psi)+\cos (\phi) \cos (\psi) \sin (\theta))+\Omega \cos (\phi) \cos (\theta) \sin (\lambda) \\
-\Omega \cos (\lambda)(\cos (\phi) \cos (\psi)+\sin (\phi) \sin (\psi) \sin (\theta))
\end{array}\right)^{T}
\end{aligned}
$$

We define $m_{\omega n}, m_{a_{x n}}$ and $m_{a_{y n}}$ as the average of observed gyro measurement data, the average of observed $\mathrm{x}$ and $\mathrm{y}$ accelerometer measurement data respectively at indexing motor positions $n=0,1,2,3$. Current residual vector in an iteration of the least squares method is denoted as $y$, and defined as the fitted value $(h(\hat{x}))$ minus the observations itself. With four indexing positions the input vector has 12 elements, $y_{1}, y_{2}, \ldots, y_{12}$. For each iteration, these are evaluated for accelerometer and gyroscope as

$$
\left(\begin{array}{l}
y_{1} \\
y_{2} \\
y_{3} \\
y_{4} \\
y_{5} \\
y_{6} \\
y_{7} \\
y_{8} \\
y_{9} \\
y_{10} \\
y_{11} \\
y_{12}
\end{array}\right)=\left(\begin{array}{c}
h_{1}-m_{a_{x 0}} \\
h_{1}+m_{a_{y 1}} \\
h_{1}+m_{a_{x 2}} \\
h_{1}-m_{a_{y 3}} \\
h_{2}-m_{a_{y 0}} \\
h_{2}-m_{a_{x 1}} \\
h_{2}+m_{a_{y 2}} \\
h_{2}+m_{a_{x 3}} \\
h_{3}+m_{\omega 1} \\
h_{3}-m_{\omega 3} \\
h_{4}-m_{\omega 0} \\
h_{4}+m_{\omega 2}
\end{array}\right) .
$$

Using these equations we can relate 12 observations to 3 unknowns (4 positions, 2 accelerometer observations and 1 gyro for each position).

For each iteration step, the shift vector $d x$ of the unknowns $x$ is computed as

$$
d x=\left(J^{T} \Sigma^{-1} J\right)^{-1} J^{T} \Sigma^{-1} y
$$

where $J$ is extended from $J_{h}$ as

$$
J=\left(\begin{array}{llllllllllll}
J_{h 1} & J_{h 1} & J_{h 1} & J_{h 1} & J_{h 2} & J_{h 2} & J_{h 2} & J_{h 2} & J_{h 3} & J_{h 3} & J_{h 4} & J_{h 4}
\end{array}\right)^{T},
$$

and $\Sigma$ is a diagonal matrix related to error variance of gyroscope and accelerometer measurements, defined in following section, and the estimate of Euler angles is updated as

$$
\hat{x}_{+}=\hat{x}-d x .
$$

Using thus estimated Euler angles the DCM (10) can be constructed. But in our method of solving the DCM we are missing the information about the $\mathrm{z}$-axis along the body frame of the instrument we used, because of which the DCM constructed using the estimated Euler angles is only one of the solution out of two possible solutions. This is because when we try to estimate the missing third axis information, using the self inner products of $w_{I B}^{B}$ and $a_{s f}^{B}$, we get

$$
\begin{gathered}
m_{a z}= \pm \sqrt{m_{a x}^{2}+m_{a y}^{2}-g^{2}}, \text { and } \\
m_{w z}= \pm \sqrt{m_{w x}^{2}+m_{w y}^{2}-\Omega^{2}}
\end{gathered}
$$

leaving us with four solutions using (7), out of which only two solutions are valid, one with both $m_{a z}$ and $m_{w z}$ to be positive, and another with both to be negative values. The other two possible solutions with opposite signs of $m_{a z}$ and $m_{w z}$ are not possible, as dictated by the inter inner product of $w_{I B}^{B}$ and $a_{s f}^{B}$.

Now with this information we can construct the second possible DCM by simply negating the terms in the DCM constructed using the estimated Euler angles, where ever the $m_{a z}$ and $m_{w z}$ terms appear as in DCM (7). Since we now have two DCM to choose from, we need a logic to find the correct one for a certain orientation estimated by the algorithm. We have found that the term $\operatorname{DCM}(3,3)$ in $(10)$, can be evaluated directly using the average of observed measurements $m_{\omega n}$, $m_{a_{x n}}, m_{a_{y n}}$ and solving equations (22) and (23). In our experiments we have used the directly evaluated $\operatorname{DCM}(3,3)$ term in (10) and compared with the two of the constructed $\operatorname{DCM}(3,3)$ terms. We chose the constructed DCM that has this term to be matching, this worked out very well in our simulation, and as well as real data tests.

\section{Error Analysis}

The diagonal matrix $\Sigma$ is used to balance the errors in sensor types. For example, assuming accelerometer measurements are more accurate than gyroscope measurements, the first 8 diagonal elements of $\Sigma$ can be a higher value of about $10^{6}$ and remaining elements to be of small value of about 4 . These values should be adjusted based on the noise properties of the 
sensors being used and indexing time. If linearization errors are neglected, the variance of errors in resulting Euler angles is $[16,(29.70)]$

$$
V(\hat{x})=\left(J^{T} \Sigma^{-1} J\right)^{-1} .
$$

Further check for additional errors (unexpected vibration, nonlinearity, sensor failure) can be obtained from residuals $v=$ $-(J d x-y)$, after bias estimates have been removed. E.g. $v=y-\hat{y}_{\text {bias }}$ if the minimum is found ( $d x$ very small). In the absence of additional errors and with the Gaussian input noise, quadratic from of the residuals $\left(v^{T} \Sigma^{-1} v\right)$ is distributed as

$$
s=v^{T} \Sigma^{-1} v \sim \chi^{2}(9) .
$$

Thus a threshold for repeating the measurement can be obtained, for example, limit $s>=22$ would require remeasuring one per cent of the trial but would protect from outliers. Our experimental results show that with larger $s$ values, the results would be incorrect.

\section{Automatic Bias Removal and Tackling of Singular Points}

To verify that any constant term has no effect in solution, we define $b$, the bias input for both accelerometer and gyroscope as

$$
b=\left(\begin{array}{llllllllllll}
m_{a x b} & -m_{a y b} & -m_{a x b} & m_{a y b} & m_{a y b} & m_{a x b} & -m_{a y b} & -m_{a x b} & -m_{\omega b} & m_{\omega b} & m_{\omega b} & -m_{\omega b}
\end{array}\right)^{T},
$$

here the \pm signs appear in the bias terms due to the $C_{B}^{B n}$ indexed rotations applied to the instrument body z-axis. The Jacobian $J$ from (32) is of the form

$$
J=\left(\begin{array}{llllllllllll}
\alpha & \alpha & \alpha & \alpha & \beta & \beta & \beta & \beta & w & w & d & d \\
0 & 0 & 0 & 0 & \gamma & \gamma & \gamma & \gamma & 0 & 0 & e & e \\
0 & 0 & 0 & 0 & 0 & 0 & 0 & 0 & v & v & f & f
\end{array}\right)^{T},
$$

where $\alpha, \beta, \gamma, w, v, d, e, f$ are the terms appearing in $\mathbf{J}$ matrix in their respective column and row positions as,

$$
\begin{aligned}
& \alpha=-g \cos (\theta), \beta=-g \sin (\phi) \sin (\theta), \gamma=g \cos (\phi) \cos (\theta), \\
& w=-\Omega \cos (\theta) \sin (\lambda)-\Omega \cos (\lambda) \cos (\psi) \sin (\theta), v=-\Omega \cos (\lambda) \cos (\theta) \sin (\psi), \\
& d=\Omega \cos (\lambda) \cos (\psi) \cos (\theta) \sin (\phi)-\Omega \sin (\lambda) \sin (\phi) \sin (\theta) \\
& e=\Omega \cos (\lambda)(\sin (\phi) \sin (\psi)+\cos (\phi) \cos (\psi) \sin (\theta))+\Omega \cos (\phi) \cos (\theta) \sin (\lambda), \\
& f=-\Omega \cos (\lambda)(\cos (\phi) \cos (\psi)+\sin (\phi) \sin (\psi) \sin (\theta))
\end{aligned}
$$

All the sensor measurements related biases can be considered to be constants, as the total time period to collect one complete set of measurements, in order to estimate the Euler angles, is just under 2 minutes. While computing $J^{T} \Sigma^{-1} y$ part of the equation (31), It can be observed that $y$ is composed of the constant terms related to sensor measurement biases (38), and $J$ as in (39), one can mathematically verify, that any constant terms such as bias will cancel out.

At few tilt angles such as close to 90 degree tilts, the proposed algorithm is faced with the problem of singular points, as the computations involve solving a linear system of equations as can be seen in (31). And this problem can be tackled by evaluating and detecting the possibility of singular point occurrence in the future iterations of the algorithm loop. If such a condition is detected, the iteration loop of the algorithm can simply be exited by outputting the current estimate of $x$ as in (33) and validating the output result with any known prior information. This prior information, for example can be previous output Euler angle estimate, and probable current estimate during the drilling process. To formulate a computationally efficient equation that can detect such a condition, we need to consider the pseudo inverse of the Jacobian matrix given by

$$
J^{\dagger}=\left(J^{T} J\right)^{-1} J^{T} .
$$

This above equation can be re-factored as

$$
J^{\dagger}=\left(t_{1}\right)^{-1}\left[\begin{array}{lll}
K_{1 T} & K_{2 T} & K_{3 T}
\end{array}\right]^{T}
$$

where

$$
\begin{aligned}
t_{1}= & +e^{2} v^{2} \gamma^{2} \alpha^{2}+e^{2} v^{2} \beta \gamma-2 d f v w \gamma^{2}+2 e f v w \beta \gamma \\
& +f^{2} w^{2} \gamma^{2}+2 f^{2} \alpha^{2} \gamma^{2}+2 v^{2} \alpha^{2} \gamma^{2} .
\end{aligned}
$$

Here we need only the term $t_{1}$, the most important and useful term of all the four re-factored terms. The remaining three terms $K_{1 T}, K_{2 T}, K_{3 T}$ are given in section $\mathrm{V}$ for readers reference. The term $t_{1}$ can be easily computed, by using the already computed elements of Jacobian matrix. This computed value can than be checked to be greater than a threshold value such as 1e-6, to avoid the algorithmic computations at neighborhoods of singular points. A careful programming of the software to avoid such singular point neighborhoods is left to the implementer of this system, for example one could follow [17, (3.10)].

\section{EXPERIMENTAL RESULTS AND DISCUSSIONS}

To test our proposed algorithm, we have used sonde, an instrument used in borehole drilling process. This sonde includes a sensor board with one axis FOG rate sensor and two axis MEMS accelerometer sensor, and a motor arrangement that turns this sensor board around 360 degrees about the longitudinal axis of the sonde body. The sensor board can be turned and locked at every 90 degree turn, which enabled us to lock at $0,90,180$ and 270 the indexed positions and take measurements. The sensor board was proprietary assembly of Geovista company, and was built in to the sonde instrument. The noise characteristics of the sensors used in our experiments were empirically evaluated using the sensor measurements. The estimated deviation (Standard deviation between 20sec averages) for the FOG sensor was $0.5^{\circ} /$ hour and the accelerometer sensor was $30 \mu \mathrm{g}$. The sonde instrument is shown in left part of Fig. 1.

To compare experimental results of the proposed algorithm with one of the recently published algorithm that follows similar indexed positional measurement data usage, we implemented the algorithm from [10]. This was straight forward to implement, using [10, Eq. 13] for tilt estimation and [10, Eq. 26] to evaluate azimuth angle, which appear to be closed from equations derived in [10, Eq. 13]. Where as our approach to solve the orientation problem is to estimate the Euler angles of the device with respect to the local horizontal plane, by applying Gauss-Newton least-square approximation method. 


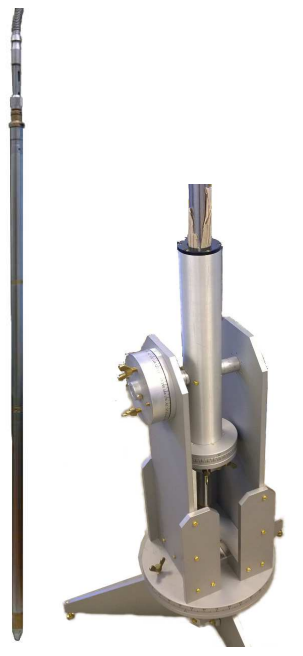

Fig. 1: Sonde instrument on the left and on the right is the jig that were used in obtaining sensor data for experiments.

In our experimental setup, a specialized 'jig' stand shown in right part of Fig. 1 was used to orient the sonde in different orientations and test the algorithms performance in estimating tilt and azimuth angles. This 'jig' has an accuracy of \pm 1 degree in orienting the sonde, this inaccuracy was not compensated for, while computing the error estimates of our proposed algorithm. Instead we assumed that the jig is perfect and can orient the sonde perfectly to required tilts and turn angles for the sake of evaluation. The sonde was tilted to a known angle about $\mathrm{x}$ axis and turned (rotated about vertical axis of 'jig') to face the azimuth at a start angle of 0 degree offset. At this stationary orientation, measurements were captured for 20 secs in each of the four indexed positions, positioned by rotating the sensor board and locking it at those indexes. These $20 \mathrm{sec}$ measurements data was averaged out for each indexed position and used to evaluate the current orientation of the sonde. At the same orientation 3 rounds of measurement and estimation were performed and reported. Then keeping the tilt angle same, we incremented the turn angles by a fixed offset steps such as $15^{\circ}$ or $30^{\circ}$ turns to complete a $360^{\circ}$ turn. At each of the offset turn angles 3 rounds of measurement and estimation were performed and reported. This whole procedure, we considered as a full cycle of a single test case. This kind of turning the sonde at fixed offset steps, will eliminate the need to have prior knowledge of the precise azimuth direction to use in the error estimation of the algorithm output. The resulting outputs in each test case should also follow the same turn offset angles as originally applied to the sonde orientation. And if there is any offset between the assumed and output azimuths, it should be constant through out the test case, and it would be the offset to the actual true north direction. We repeated such test cases by changing the tilt angles and following the above mentioned procedure. The different orientations of the sonde in each test case using the actual sonde at $53.26 \mathrm{deg}$ latitude in UK, are depicted in Fig. 2.

Using thus collected measurement data, we have tested both proposed least-square algorithm and algorithm from
[10, Eq. 13] and obtained the azimuth and tilt estimates w.r.t horizontal plane as shown in Figs. 3, 4, 5, and 6. Figs. 3(a), 4(a), 5(a), and 6(a) shows azimuth solution in upper half part and azimuth estimate error in the bottom half parts. From these figures, it can be clearly seen that our proposed algorithm estimates azimuth very well. Left half parts of Figs. 3(b), 4(b), 5(b), and 6(b), shows the tilt estimate errors of both the algorithms, and the right half parts show the results of our proposed algorithm alone along with the measurement reliability indicator value $s$, evaluated as mentioned in (37). Couple of outliers in the tilt estimate errors can be observed from the tilt estimate error figures. The corresponding large $s$ values of these outliers indicate that the measurement data is unreliable and calls for re-measurement of those particular sample measurement instances.

We have performed additional tests on the proposed algorithm by simulating the test cases for different orientations and latitudes, as it was practically not possible to test with the actual sonde at all different locations on earth. In these simulations, measurement data was obtained for different orientations and at different latitudes and with the same noise characteristics as of the real sonde sensors. By applying the logic of thresholding the $t_{1}(42)$ in our implementations, we could avoid all the possible calculations at the neighborhoods of singular points, and found out from our simulation results, that our proposed algorithm works for almost all the orientations at all the latitudes with just one exception. The exception exists at the precise latitudes of $\pm 90^{\circ}$, as can be clearly observed from the algorithmic equations and also at these latitudes, is the earths axis of rotation and the true north coincides with $\mathrm{Z}$ axis of the horizontal plane itself. Normally there would not be a need to estimate the Euler angles at these precise latitudes. Another issue we noticed at $90^{\circ}$ tilt angles at any given latitude was that, choosing correct one out of the two possible DCMs is difficult, as the numerical calculations of $\operatorname{DCM}(3,3)$ approach zero. In this case we always had one of the DCM solution as the correct one. So this case would need further investigation which is left for our future work. The results of simulation tests compared to [10], are provided in Table I, it includes zero degree and 4 degree tilt cases where [10] algorithm is seen to be working at its best.

The Azimuth RMS error of these tests was a max 2.6 degrees with a tilt RMS error of 0.6 degrees with all the test cases performed with our proposed algorithm, where as algorithm from [10, Eq. 13] fails when the angle of tilts are greater than 5 degrees. As this was one of the many algorithms that were possibly meant to be used when the instruments sensitive axis is strapped down in perpendicular orientation w.r.t horizontal plane rather than at an arbitrary angle. The results of the proposed algorithm can be improved further by analyzing the FOG characteristics more closely, for example, some of the FOG noise characteristic analysis methods presented in [18] could be used to analyze and optimize the sampling time and improve the accuracy of azimuth estimate further. But in real time systems where the azimuth should be estimated in matter of shortest possible time, tradeoff between accuracy and time to azimuth fix should be made. There are other important error sources that contribute to the azimuth 


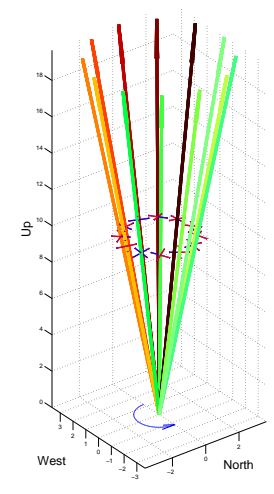

(a) 10 deg tilt

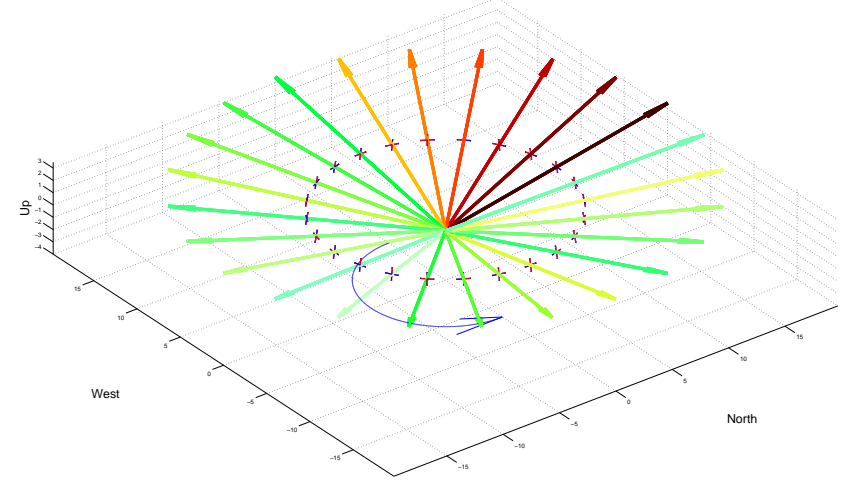

(c) 80 deg tilt

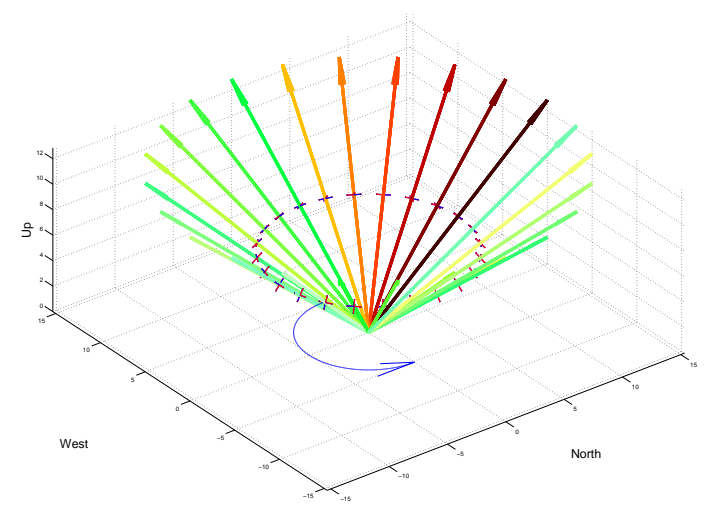

(b) 50 deg tilt

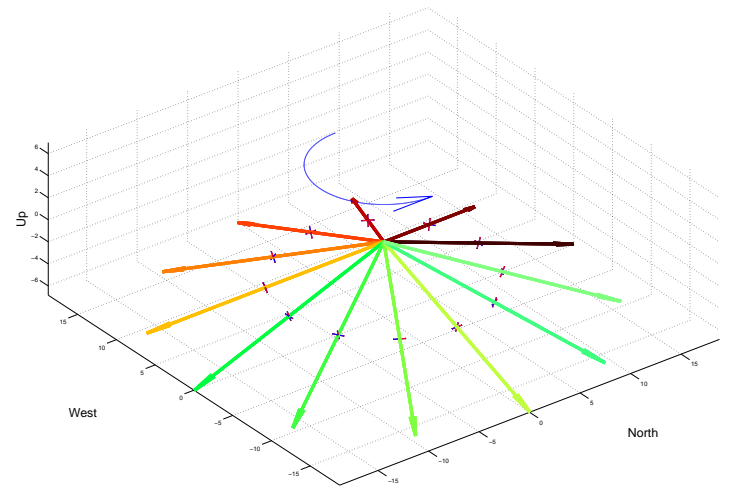

(d) 110 deg tilt

Fig. 2: Different orientations of the sonde instrument during the measurements. Sonde facing with azimuth starts at 0 deg and incremented at 15/30 degrees to make one round of 360 degrees. The + marks in the middle of the sonde indicates the approximate location of the sensor board and corresponding $\mathrm{X}, \mathrm{Y}$ axis.

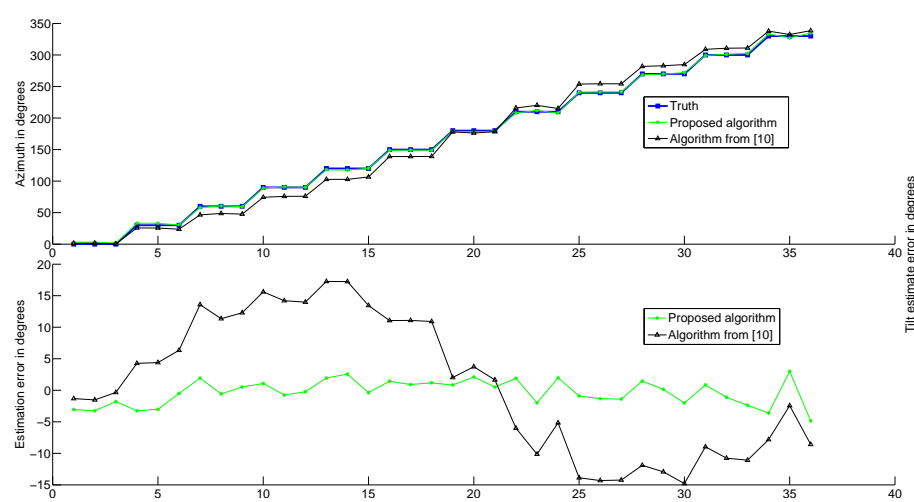

(a)

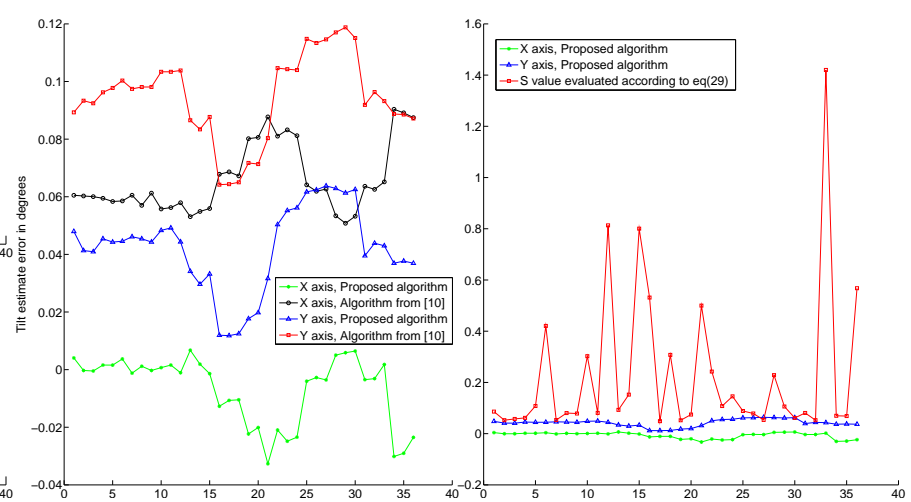

(b)

Fig. 3: Comparison between the proposed algorithm and [10] with 10 deg tilt about Y axis. (a) Azimuth estimate top and estimate error bottom and (b) Error in tilt estimate, both algorithms on left and proposed algorithm along with value of measurement reliability indicator $S$ on the right. 


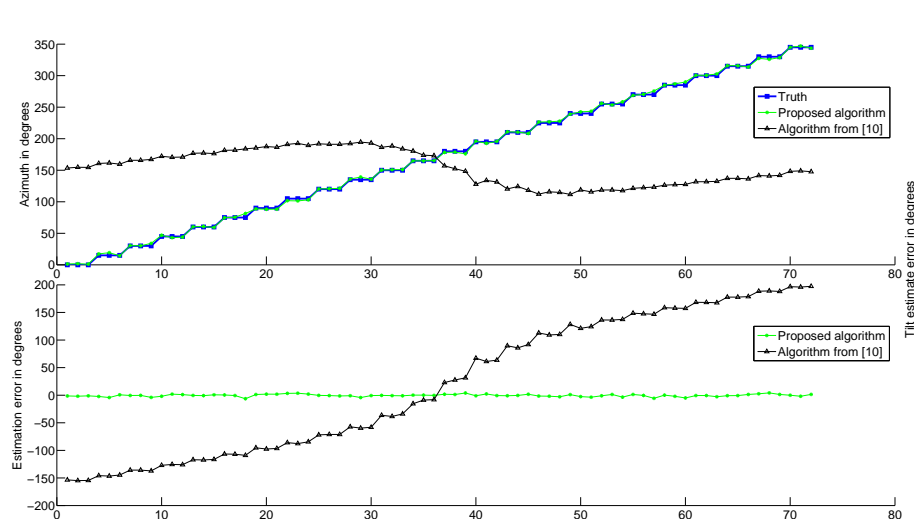

(a) Azimuth estimate top and estimate error bottom
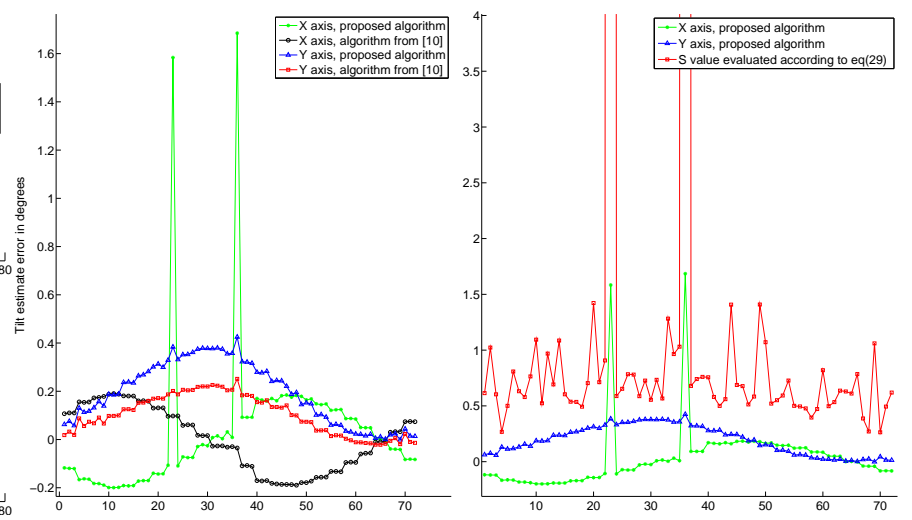

(b) Error in tilt estimate, both algorithms on left and proposed algorithm along with value of measurement reliability indicator $S$ on the right

Fig. 4: Comparison between the proposed algorithm and [10] with 50 deg tilt about Y axis.

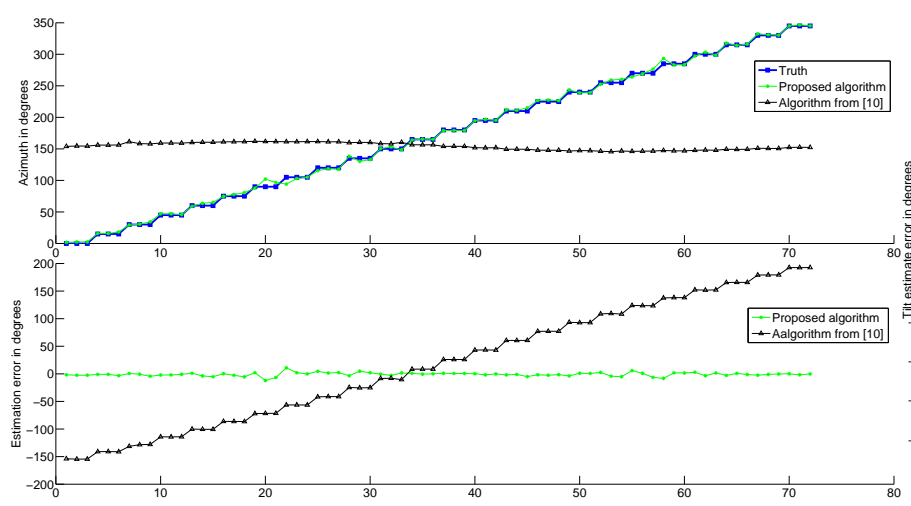

(a) Azimuth estimate top and estimate error bottom
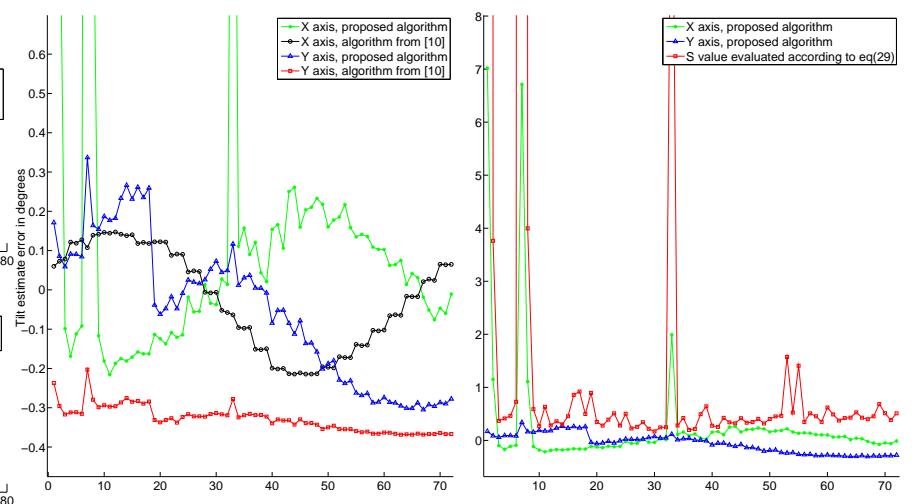

(b) Error in tilt estimate, both algorithms on left and proposed algorithm along with value of measurement reliability indicator $S$ on the right

Fig. 5: Comparison between the proposed algorithm and [10] with 80 deg tilt about $\mathrm{Y}$ axis.

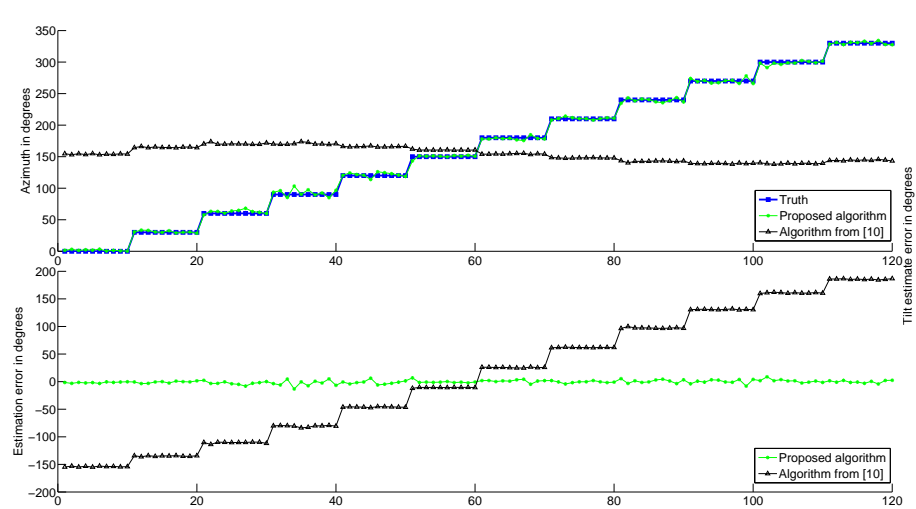

(a) Azimuth estimate top and estimate error bottom
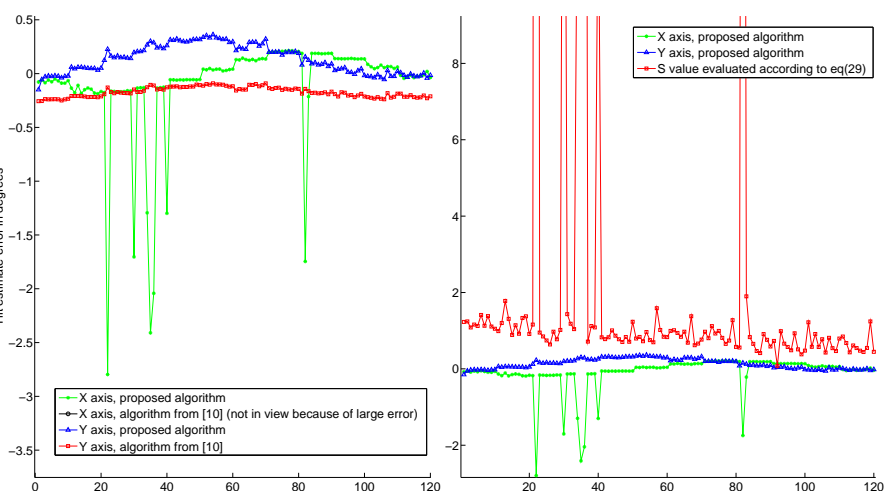

(b) Error in tilt estimate, both algorithms on left and proposed algorithm along with value of measurement reliability indicator $S$ on the right

Fig. 6: Comparison between the proposed algorithm and [10] with 110 deg tilt about Y axis. 
TABLE I: Experiment Results with Simulated Measurement Data at $60.5^{\circ}$ Latitude.

All units are in degrees.

\begin{tabular}{|c|c|c|c|c|c|c|c|c|c|}
\hline \multicolumn{3}{|c|}{ True Orientation } & \multicolumn{4}{|c|}{ Estimation Error of Proposed Algorithm } & \multicolumn{3}{|c|}{ Estimation Error of [10] } \\
\hline Azimuth & Roll/Tilt1 & Pitch/Tilt2 & Azimuth & Roll/Tilt1 & Pitch/Tilt2 & Meas.Rel 'S' & Azimuth & Roll/Tilt1 & Pitch/Tilt2 \\
\hline 0 & 0 & 0 & -2.2419 & $6.5135 \mathrm{e}-06$ & $2.8574 \mathrm{e}-06$ & 4.1324 & -2.2416 & $-3.1081 \mathrm{e}-05$ & $1.0718 \mathrm{e}-05$ \\
\hline 30 & 0 & 0 & 1.5603 & $4.3562 \mathrm{e}-05$ & $2.4615 \mathrm{e}-05$ & 6.6876 & 1.5603 & 0.0002222 & $5.2856 \mathrm{e}-05$ \\
\hline 60 & 0 & 0 & -0.7294 & $9.4943 e-05$ & $6.8265 \mathrm{e}-05$ & 7.5515 & -0.7292 & 0.0001412 & 0.0001603 \\
\hline 90 & 0 & 0 & 0.0871 & $7.4497 \mathrm{e}-05$ & -0.0001005 & 6.7578 & 0.0870 & 0.0001449 & $-3.1338 \mathrm{e}-05$ \\
\hline 120 & 0 & 0 & -2.0852 & $2.7409 \mathrm{e}-05$ & $4.3059 \mathrm{e}-05$ & 4.4622 & -2.0852 & $2.7338 \mathrm{e}-05$ & $9.7869 \mathrm{e}-05$ \\
\hline 150 & 0 & 0 & -2.8262 & $-6.5587 \mathrm{e}-05$ & $7.7639 \mathrm{e}-05$ & 3.2442 & -2.8262 & $-4.3061 \mathrm{e}-05$ & $2.1923 \mathrm{e}-05$ \\
\hline 180 & 0 & 0 & 0.8234 & $-1.3132 \mathrm{e}-05$ & $3.6996 \mathrm{e}-05$ & 6.1216 & 0.8236 & $1.4234 \mathrm{e}-06$ & $4.8226 \mathrm{e}-06$ \\
\hline 210 & 0 & 0 & 1.8452 & $-4.2683 \mathrm{e}-05$ & $6.6364 \mathrm{e}-05$ & 4.6476 & 1.8453 & $-7.5048 \mathrm{e}-07$ & 0.0001754 \\
\hline 240 & 0 & 0 & -2.6937 & $5.4557 \mathrm{e}-05$ & $-1.4757 \mathrm{e}-06$ & 4.0023 & -2.6935 & $1.3175 \mathrm{e}-05$ & $-7.6701 \mathrm{e}-05$ \\
\hline 270 & 0 & 0 & -0.9897 & $-6.6461 e-06$ & $5.2205 \mathrm{e}-05$ & 3.0918 & -0.9897 & $3.2492 \mathrm{e}-05$ & $-2.1222 \mathrm{e}-05$ \\
\hline 300 & 0 & 0 & 0.3133 & $-5.3306 e-05$ & $9.5496 \mathrm{e}-07$ & 3.3843 & 0.3135 & $-2.7965 e-05$ & $1.7729 \mathrm{e}-05$ \\
\hline 330 & 0 & 0 & 0.7973 & $6.6273 e-05$ & 0.0001308 & 1.5632 & 0.7973 & $4.8899 \mathrm{e}-05$ & 0.0001439 \\
\hline 0 & 4 & 0 & -0.3079 & $7.1357 \mathrm{e}-05$ & $7.6276 e-05$ & 2.2842 & -0.3543 & 0.0002 & $6.9228 \mathrm{e}-05$ \\
\hline 30 & 4 & 0 & -0.8906 & $-2.0648 \mathrm{e}-05$ & $-0.9403 e-05$ & 7.4073 & -4.9733 & -0.0001 & $4.6142 \mathrm{e}-05$ \\
\hline 60 & 4 & 0 & -1.4108 & $-8.9556 e-05$ & $2.8552 \mathrm{e}-05$ & 3.3591 & -8.3423 & -0.0001 & $3.9290 \mathrm{e}-05$ \\
\hline 90 & 4 & 0 & -1.3241 & $2.2140 \mathrm{e}-05$ & $2.0888 \mathrm{e}-05$ & 4.8748 & -9.2816 & $-3.6107 e-05$ & $3.4907 \mathrm{e}-05$ \\
\hline 120 & 4 & 0 & 0.3459 & $-1.8640 \mathrm{e}-05$ & $5.2456 \mathrm{e}-05$ & 4.9107 & -5.5267 & $1.0872 \mathrm{e}-05$ & -0.0001 \\
\hline 150 & 4 & 0 & 0.0247 & $-2.9805 \mathrm{e}-05$ & $1.2839 \mathrm{e}-05$ & 2.3191 & -3.1669 & $-1.3887 \mathrm{e}-05$ & 0.0001 \\
\hline 180 & 4 & 0 & -0.2862 & $1.6880 \mathrm{e}-05$ & $6.3853 e-05$ & 6.3010 & -0.2537 & $1.5954 \mathrm{e}-05$ & $2.4349 \mathrm{e}-05$ \\
\hline 210 & 4 & 0 & 0.4822 & $7.8378 \mathrm{e}-06$ & $2.9041 \mathrm{e}-05$ & 2.4946 & 3.6190 & -0.0001 & $1.3303 \mathrm{e}-05$ \\
\hline 240 & 4 & 0 & -0.8927 & $3.1241 \mathrm{e}-05$ & $-2.8380 \mathrm{e}-05$ & 4.2681 & 5.3932 & $-3.2187 e-05$ & $6.3404 \mathrm{e}-05$ \\
\hline 270 & 4 & 0 & -1.6420 & $-8.0813 e-05$ & $7.0152 \mathrm{e}-05$ & 6.5392 & 5.7581 & $1.3400 \mathrm{e}-05$ & $8.8615 \mathrm{e}-05$ \\
\hline 300 & 4 & 0 & -1.5996 & $7.4185 \mathrm{e}-05$ & $7.1517 \mathrm{e}-05$ & 3.6179 & 4.3924 & $3.1925 \mathrm{e}-05$ & $4.5624 \mathrm{e}-05$ \\
\hline 330 & 4 & 0 & -2.1553 & $-3.3062 \mathrm{e}-06$ & $-4.1540 e-05$ & 4.9186 & 1.8530 & $2.4348 \mathrm{e}-05$ & $-3.9768 \mathrm{e}-05$ \\
\hline
\end{tabular}

and tilt estimates, for example [19] et al. have studied and presented error analysis report for gyro North seeking systems using DCM. They have found that plumb errors of spindle axis, angular rate accuracy of gyro, measurement accuracy of angles, and latitude, all have influence on final measurement accuracy of azimuth estimation. In addition, few important steps such as, setting correct latitude, verifying strength of local gravity in new location and allowing some minutes for the device to warm up, should be taken care before using the system. Scale factor of the gyroscope should also be calibrated, as scale factor errors lead to azimuth bias.

\section{CONCLUSIONS}

In this article, we have proposed a novel algorithm to find the orientation of an object strapped down to earth, with respect to true North and earth's horizontal plane. It requires only one single-axis FOG rate sensor and one two-axes MEMS accelerometer. The algorithm was tested for its capability to estimate the azimuth and tilts, with the equipment tilted in almost all the angles ranging from $0^{\circ}$ to $\pm 90^{\circ}$ about the local vertical. The accuracy of the proposed algorithm was evaluated to be $2.6^{\circ} \max \mathrm{RMS}$ azimuth error and $0.6^{\circ}$ of $\max \mathrm{RMS}$ tilt error with the sensors that we used. This could further be improved by additional methods mentioned in this publication. The proposed algorithm is applicable for situations where knowing the true North and inclination is important, such as mining industries, petroleum industries, borehole surveying and environmental research work.

\section{Appendix: Refactored Pseudoinverse of J}

The re-factored pseudoinverse of $J^{\dagger}$ was given in (41), and the remaining three terms $K_{1 T}, K_{2 T}, K_{3 T}$ are given as:

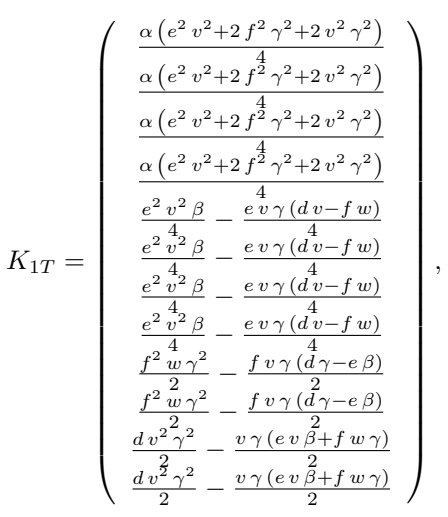

and

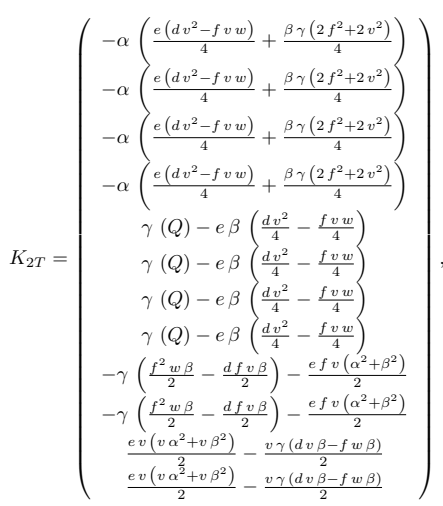

where

$Q=\frac{d^{2} v^{2}}{4}-\frac{d f v w}{2}+\frac{f^{2} w^{2}}{4}+\frac{f^{2} \alpha^{2}}{2}+\frac{v^{2} \alpha^{2}}{2}$, 
and

$$
K_{3 T}=\left(\begin{array}{c}
-\alpha\left(\frac{f\left(2 d \gamma^{2}-2 e \beta \gamma\right)}{4}+\frac{v w\left(e^{2}+2 \gamma^{2}\right)}{4}\right) \\
-\alpha\left(\frac{f\left(2 d \gamma^{2}-2 e \beta \gamma\right)}{4}+\frac{v w\left(e^{2}+2 \gamma^{2}\right)}{4}\right) \\
-\alpha\left(\frac{f\left(2 d \gamma^{2}-2 e \beta \gamma\right)}{4}+\frac{v w\left(e^{2}+2 \gamma^{2}\right)}{4}\right) \\
-\alpha\left(\frac{f\left(2 d \gamma^{2}-2 e \beta \gamma\right)}{4}+\frac{v w\left(e^{2}+2 \gamma^{2}\right)}{4}\right) \\
-v\left(\frac{e^{2} w \beta}{4}-\frac{d e w \gamma}{4}\right)-\frac{e f \gamma\left(w^{2}+2 \alpha^{2}\right)}{4} \\
-v\left(\frac{e^{2} w \beta}{4}-\frac{d e w \gamma}{4}\right)-\frac{e f \gamma\left(w^{2}+2 \alpha^{2}\right)}{4} \\
-v\left(\frac{\left.\frac{e^{2} w \beta}{4}-\frac{d e w \gamma}{4}\right)-\frac{e f \gamma\left(w^{2}+2 \alpha^{2}\right)}{4}}{4}\right) \\
-v\left(\frac{e^{2} w \beta}{4}-\frac{d e w \gamma}{4}\right)-\frac{e f \gamma\left(w^{2}+2 \alpha^{2}\right)}{4} \\
R-f w\left(\frac{d \gamma^{2}}{2}-\frac{e \beta \gamma}{2}\right) \\
R-f w\left(\frac{d \gamma^{2}}{2}-\frac{e \beta \gamma}{2}\right) \\
\frac{f \gamma\left(\gamma w^{2}+\gamma \alpha^{2} 2\right)}{2}-\frac{v \gamma(d w \gamma-e w \beta)}{2} \\
\frac{f \gamma\left(\gamma w^{2}+\gamma \alpha^{2} 2\right)}{2}-\frac{v \gamma(d w \gamma-e w \beta)}{2}
\end{array}\right),
$$

where

$$
R=v\left(\frac{d^{2} \gamma^{2}}{2}-d e \beta \gamma+\frac{e^{2} \alpha^{2}}{2}+\frac{e^{2} \beta^{2}}{2}+\alpha^{2} \gamma^{2}\right) .
$$

\section{REFERENCES}

[1] J. Barnes, L. Cheng, and K. Ariyur, "A hemispherical sun sensor for orientation and geolocation," Sensors Journal, IEEE, vol. 14, no. 12, pp. 4423-4433, Dec 2014.

[2] I. P. Prikhodko, S. A. Zotov, A. A. Trusov, and A. M. Shkel, "What is mems gyrocompassing? Comparative analysis of maytagging and carouseling," Microelectromechanical Systems, vol. 22, no. 6, pp. 12571266, Dec 2013.

[3] S. Jun, M. Lingjuan, G. Weixi, and S. Jing, "Design and realization of low-cost, fast and high-precision fog north finder," in Electrical and Control Engineering (ICECE), 2010 International Conference on, June 2010, pp. 695-698.

[4] R. Mahony, T. Hamel, and J.-M. Pflimlin, "Nonlinear complementary filters on the special orthogonal group," Automatic Control, IEEE Transactions on, vol. 53, no. 5, pp. 1203-1218, June 2008.

[5] S. Madgwick, A. Harrison, and R. Vaidyanathan, "Estimation of IMU and MARG orientation using a gradient descent algorithm," in Rehabilitation Robotics (ICORR), 2011 IEEE International Conference on, June 2011, pp. 1-7.

[6] P. Savage, "Strapdown inertial navigation integration algorithm design," Journal of Guidance, Control and Dynamics, vol. 21, no. 1-2, 1998.

[7] W. S. Watson, "Improved north seeking gyro," in Position Location and Navigation Symposium, 1992. Record. 500 Years After Columbus Navigation Challenges of Tomorrow, 1992, pp. 121-125.

[8] T. Tomohiro, I. Yuichi, N. Makoto, and Y. Toshihiko, "Automatic north sensor using a fiber-optic gyroscope," Appl. Opt., vol. 33, no. 1, pp. 120-123, Jan 1994.

[9] L. I. Iozan, M. Kirkko-Jaakkola, J. Collin, J. Takala, and C. Rusu, "Using a mems gyroscope to measure the earths rotation for gyrocompassing applications," Measurement Science and Technology, vol. 23, no. 2, p. $025005,2012$.

[10] S. Hua, Z. Fang, and L. Haojun, "Design and implementation of fiber optic gyro north-seeker," in Mechatronics and Automation (ICMA), 2010 International Conference on, Aug 2010, pp. 1058-1062.

[11] G. Wahba, "A least squares estimate of spacecraft attitude," SIAM Review, vol. 7, no. 3, p. 409, July 1965.

[12] F. L. Markley, "Attitude determination using vector observations and the singular value decomposition," The Journal of the Astronautical Sciences, vol. 36, no. 3, pp. 245-258, 1988.

[13] A. Saxena, G. Gupta, V. Gerasimov, and S. Ourselin, "In use parameter estimation of inertial sensors by detecting multilevel quasi-static states," in Knowledge-Based Intelligent Information and Engineering Systems, ser. Lecture Notes in Computer Science, R. Khosla, R. Howlett, and L. Jain, Eds. Springer Berlin Heidelberg, 2005, vol. 3684, pp. 595601.

[14] Y. F. Jiang, "Error analysis of analytic coarse alignment methods," Aerospace and Electronic Systems, IEEE Transactions on, vol. 34, no. 1, pp. 334-337, Jan 1998.

[15] D. H. Titterton and J. L. Weston, Strapdown Inertial Navigation Technology, 2nd ed., ser. American Institute of Aeronautics, Reston, VA, P. Z, Ed. Strapdown Associates, Inc., 2004, vol. 207.

[16] A. Stuart and K. Ord, Kendall's Advanced Theory of Statistics, ser. Kendall's Advanced Theory of Statistics. Wiley, 2009, vol. 1: Distribution Theory, no. v. 1; v. 1994.
[17] Radhakrishna, Linear Models: Least Squares and Alternatives. Second Edition, ser. Springer Series in Statistics. Springer Publishing, 1999.

[18] H. Changhong, Y. Chuanchuan, W. Xinyue, and W. Ziyu, "Enhanced multiposition method to suppress the north finding error caused by bias drift with fiber optic gyroscopes," Appl. Opt., vol. 52, no. 21, pp. 53035311, Jul 2013.

[19] R. Shunqing, Z. Zhenhao, and C. Yan, "Error analysis for the gyro north seeking system," in Systems and Control in Aerospace and Astronautics, 2006. ISSCAA 2006. 1st International Symposium on, Jan 2006, pp. 4786.

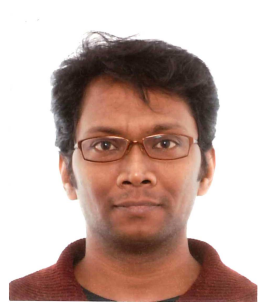

Jayaprasad Bojja received Bachelors of Engineering in Computer Science and Engineering from Osmania University, Hyderabad, India in 1998. Since then he has been working in Software Industry in different roles, the last role before moving on to higher studies in 2009 was, Team lead at Wipro Technologies and Ext-Technical Consultant at Nokia Finland.

During the period of higher studies, he has worked as researcher at Tampere University of Technology (TUT), Tampere, Finland. He has received the degree of M.Sc. in Multimedia Signal Processing in 2011 with Honours, from TUT. He is finalizing his Ph.D. studies and currently working with Space Systems Finland.

His professional interests include, software engineering, algorithms, artificial Intelligence, positioning and navigation, and applications involving any of the methods and techniques such as statistics, multimedia signal processing, sensors signal processing, pattern recognition.

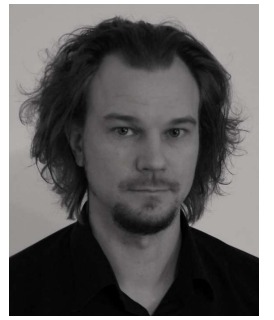

Jussi Collin (M'11) received the M.Sc. and Dr.Tech. degrees from the Tampere University of Technology, Tampere, Finland, in 2001 and 2006, respectively, specializing in sensor-aided personal navigation.

$\mathrm{He}$ is currently Senior Research Fellow and Adjunct Professor at Department of Pervasive Systems, Tampere University of Technology.

His research interests include statistical signal processing and novel sensor-based navigation applications.

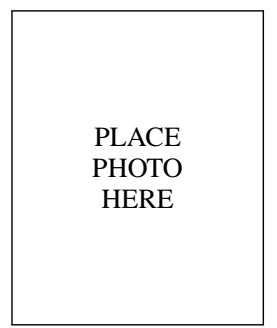

Martin Payne is with Geovista Ltd, Glan Convy, UK.

Ryan Grifths is with Geovista Ltd, Glan Convy, UK.

PLACE

PHOTO

HERE 


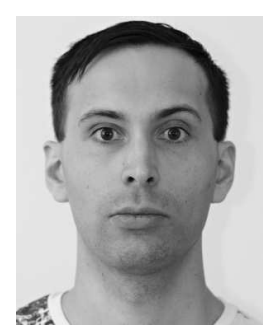

Martti Kirkko-Jaakkola received his M.Sc.(tech.) and D.Sc.(tech.) degrees from Tampere University of Technology, Tampere, Finland, in 2008 and 2013, respectively.

From 2006 to 2013 he was with the Department of Pervasive Computing, Tampere University of Technology. Currently, he is a Senior Research Scientist at the Finnish Geospatial Research Institute, National Land Survey of Finland, in Kirkkonummi, Finland. His research interests include low-cost inertial sensors and precise satellite positioning.

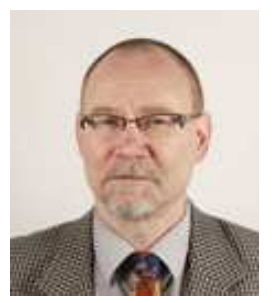

Jarmo Takala received his M.Sc. (hons) degree in Electrical Engineering and Dr.Tech. degree in Information Technology from Tampere University of Technology, Tampere, Finland (TUT) in 1987 and 1999 , respectively.

From 1992 to 1995 , he was a Research Scientist at VTT-Automation, Tampere, Finland. Between 1995 and 1996, he was a Senior Research Engineer at Nokia Research Center, Tampere, Finland. From 1996 to 1999 , he was a Researcher at TUT. Since 2000, he has been Professor in Computer Engineering at TUT and currently Dean of the Faculty of Computing and Electrical Engineering of TUT. Dr. Takala is Co-Editor-in-Chief for Springer Journal on Signal Processing Systems. During 2007-2011 he was Associate Editor and Area Editor for IEEE Transactions on Signal Processing and in 2012-2013 he was the Chair of IEEE Signal Processing Society's Design and Implementation of Signal Processing Systems Technical Committee. His research interests include circuit techniques, parallel architectures, and design methodologies for digital signal processing systems. 